(IMF)/CESR Fellowships) in the United Kingdom and to understand shared themes.

Method. Three psychiatrists with the experience of being part of MTI/IMF program, for a minimum of 1 year, participated in theme guided, focussed discussions to understand common experiences. These discussion where limited to 3 broad headings. Opportunities to grow, what we wish the college knew and what we wished the trusts and supervisors knew. The experiential accounts were captured and circulated among a group of 20 MTI/IMF/CESR fellowship doctors and rated on a 5 point Likert scale varying between strongly agree to strongly disagree.

Result. The findings suggest that the expectations and experiences of the psychiatrists on such programs share some common themes. Most of them had varied experiences under the theme 'opportunities to grow'. The suggestions for what these doctors 'wished the trusts, college and supervisors knew' had a good concordance among the 20 doctors who reviewed the themes and suggestions. The details of the themes and commonalities will be discussed at the conference.

Conclusion. The expectations and experiences of the doctors on MTI/equivalent program share common themes. Bridging the gap between MTI experience to an excellent MTI experience would involve identifying such shared experiences, that could potentially guide development of processes, thereby making these training fellowships better tailored to each trainee.

\section{Regional survey into trainee experience of core psychotherapy training}

Lauren Evans ${ }^{1 \star}$ and Georgia Belam ${ }^{2}$

${ }^{1}$ South West London and St George's Mental Health NHS Trust and

${ }^{2}$ Surrey and Borders Partnership NHS Foundation Trust

${ }^{*}$ Corresponding author.

doi: 10.1192/bjo.2021.386

Aims. This project aims to assess the experience of psychiatry core trainees who have undertaken core psychotherapy training (CPT), to identify what is experienced positively and potential areas of improvement.

Background. Psychotherapy is an necessary part of core psychiatry training, requiring one short and one long case to complete core training.

Method. An anonymous online survey was drafted, containing both qualitative and quantitative questions, to assess trainees experiences of CPT. It was circulated via Trust email (locally) and Twitter (nationally).

Result. A total of 35 responses were received: 21 core trainees, 12 higher trainees, 1 consultant, and 1 staff grade doctor. 6 respondents had completed a short case only; 2 a long case only; 25 both; and 2 neither.

Confidence in psychotherapy knowledge was rated on a 1-5 scale (1: significantly below average to 5 : significantly above average). Theoretical knowledge improved from a 2.57 average before $\mathrm{CPT}$ to 3.63 following, and clinical application improved from 2.43 before to 3.66 following.

Knowledge prior to delivering CPT was most commonly obtained from Balint group (71.4\% of respondents) and MRCPsych courses (65.7\%).

The main barriers to obtaining psychotherapy experience were: accessing supervision (60.0\% of respondents); not enough patients (53.3\%); and a lack of guidelines on accessing supervision and patients (43.3\%). Additionally, getting time away from day jobs was identified as a concern, particularly among LTFT trainees.

Important learning points from CPT identified by trainees were: knowledge of psychoanalytic concepts, such as transference and counter-transference; differences between the theoretical models; an alternative approach to formulation; and how these skills can be useful in all clinical encounters, such as maintaining rapport, boundaries, and time-keeping. The useful role of supervision was also highlighted.

Conclusion. This project serves as an introductory look into how trainees view their experience of CPT, and potential areas for improvement.

Themes for improvement, arising from qualitative responses, are: clear reading list, including introductory materials; clear guidelines at induction, including supervisor contact details; improved access to supervision; patients to be allocated; protected time for psychotherapy, with extra support for LTFTs; shadowing; increased choice of modality; and more formal teaching on psychotherapy. These are key areas to be targeted to improve the trainee experience, particularly for those who risk delays in their training.

\section{West Midlands general adult psychiatry higher trainees' peer group wellbeing away day}

\section{Emma Fisher*}

Coventry and Warwickshire Partnership Trust ${ }^{\star}$ Corresponding author.

\section{doi: 10.1192/bjo.2021.387}

Aims. The main aim of the wellbeing day was to increase the sense of wellbeing amongst psychiatry higher trainees in the West Midlands. We first wanted to understand the wellbeing needs of the trainees and what they hoped to get out of an away day. We wanted then to evaluate whether the away day had met these needs. Background. The Psychiatry Trainees Committee (PTC) published a report 'Supported and Valued? A trainee led review into morale and training within psychiatry' in 2017. The importance of feeling valued and supported and the value trainees place upon the support of their peers, were highlighted in this report.

As higher trainees we are often geographically isolated from each other, and whilst the peer group meet once per month, this is mostly for academic lectures resulting in poor familiarity amongst trainees which can leave trainees feeling unknown and unsupported.

Method. We decided to apply to HEE for funding for an away day. We surveyed the peer group, asking what they most wanted to get out of an away day. The results showed that 'a morale boost', 'destress/relaxation' and 'opportunity to get to know other trainees' were the trainee's priorities, followed by improving leadership, team working and negotiation skills.

With these priorities in mind, an away day programme was developed which included a talk from Dr Mike Blaber, a palliative care doctor with a special interest in doctors' wellbeing, a 'getting to know you' art activity and a team building GPS treasure hunt funded by HEE. The day finished with a dinner in a local restaurant sponsored by Recordati. The rest of the day was paid for by the peer group.

Result. 28 higher trainees attended the away day which was held in Birmingham on 11/07/2019. Trainees gave feedback on the day using an online anonymous survey. $81 \%$ of attendees said the away day decreased their stress levels. $90 \%$ said that the day had increased their sense of wellbeing. $86 \%$ felt an increased sense of belonging and less isolated as a trainee.

Conclusion. Regular trainee away days may help tackle isolation and increase morale which is linked to better patient outcomes. Improving trainees' sense of wellbeing leads to better job satisfaction, which may ultimately lead to higher rates of retention within psychiatry. 